\title{
Advanced flow cell design for in vitro release testing of mucoadhesive buccal films
}

BLAŽ GRILC*

JURIJ ZDOVC

ODON PLANINŠEK

University of Ljubljana, Faculty of Pharmacy, Chair of Pharmaceutical Technology, 1000 Ljubljana, Slovenia
Accepted October 25, 2019

Published online November 18, 2019
Films for buccal application are a slowly emerging new platform for drug delivery. There remains a lack of analytical techniques for the determination of in vitro active pharmaceutical ingredient release. The aim here was to develop an alternative method to the commonly used United States Pharmacopoeia (USP) 2 method, based on the flow-through cell. This system extends the release time and enables more detailed sample discrimination according to formulation. It could be used as a tool for in vivo prediction of drug release rates from buccal film formulations. The flow cell contains two chambers separated by a membrane through which the released active pharmaceutical ingredient is measured. Vital system variables and their effects on the release rate of the model active pharmaceutical ingredient are presented for formulations based on sodium alginate polymer. The method reflects the differences between films and is shown to be discriminatory for evaluation of buccal formulations.

Keywords: release rate, buccal films, hydrostatic pressure, flow cell

The oral route for drug delivery is the most desired way of drug administration in terms of patient compliance (1). Orally dispersible formulations that are easy to apply or act as instant release are being improved in terms of tablets and thin films. Indeed, films for the buccal application have been introduced and are gaining in popularity (2). Films can be adhesive to the mucosa and provide rapid drug release, with the drug absorbed from the oral cavity directly into the blood system $(3,4)$. The time between drug administration and effect taking place can be shortened and the first-pass metabolism can be avoided. Films are administered without water and are suitable for patients who are suffering from dysphagia $(5,6)$. Films can be composed of multiple layers and facilitate drug release from both or only one side $(7,8)$. However, because of their unique properties, films are challenging for development and manufacture. There is a lack of suitable in vitro dissolution testing techniques for researchers to evaluate formulation's drug release rate properties (9). Research and development of films comprise a balance between appearance, mechanical properties, and efficacy of the final product (10).

\footnotetext{
*Correspondence, e-mail: blaz.grilc@ffa.uni-lj.si
} 
Release testing is a common tool to evaluate the properties of films in vitro (11). Many dissolution procedures have been described in pharmacopoeias, but none of these are suitable for film formulations (12). Conventional methods cause films to dissolve in a few minutes when there are large volumes of the dissolution medium and good liquid flow. Comparing the active pharmaceutical ingredient release profiles of two dissolving films typically does not provide discriminatory data, for example due to different composition. Therefore, the effect of the changing formulation composition on the drug release profile is difficult to assess.

In the present study, a method to extend release times and to express differences between film formulations was developed. Extending the release times helps to distinguish the formulations with a faster drug release rate. Release testing systems for films that are an alternative to pharmacopoeia tests have been introduced in many studies. Xia et al. introduced a flow-through cell (12), where the film was positioned in the chamber of a flow cell. A constant flow of dissolution medium was maintained using a syringe pump. The samples eluted from the flow cell were collected and the drug release was quantified. Aliaa et al. used the US Pharmacopoeia (USP) apparatus II with the film fixed on a glass slab, to avoid floating of the formulation (13). The glass slab was used as a sinker and support, with the whole structure placed at the bottom of the dissolution vessel.

Krampe et al. proposed a release method that used a filter frame in which the film was placed and pressed to the filter using a punch (14). This whole structure, including the punch, was placed in the USP apparatus II vessel and it floated on the surface of the dissolution medium. During mixing by the paddle, the drug release was determined at different times. Laitinen et al. proposed a release method that used a flow cell in which the film was placed (15). The dissolution medium was pumped in a closed loop through the cell using a peristaltic pump. Here, drug release was detected by reflectance measurements with a He-Ne laser beam that passed through the glass walls of the flow cell. There was thus no sampling needed, and the dissolution volume remained the same.

Adrover and Nobili (16) and Adrover et al. (17) introduced a millifluidic flow-through device for film release testing. This consisted of a small flow cell $(2 \mathrm{~mm} \times 2.2 \mathrm{~cm} \times 3 \mathrm{~cm})$, a medium reservoir and a volumetric pump. The oral thin film was placed on the wall of the cell and was exposed to the dissolution medium from one side only. The medium was passed through the cell at a flow rate of 2 to $20 \mathrm{~mL} \mathrm{~min}^{-1}$, with samples taken from the eluted medium and analysed separately.

For all of these release rate techniques, only one side of the film was wetted during the testing. However, under in vivo conditions, the film is wetted from both sides. In the oral cavity, constant moisture is present and causes swelling of the polymer. Therefore, the film swells and erodes from the mucosa and the oral cavity side. In the present study, the discriminatory method facilitates the release rate of buccal films from both sides.

\section{EXPERIMENTAL}

\section{Materials}

Na-alginate protanal 10/60 was purchased from FMC Health and Nutrition (USA). Tartrazine was purchased from Sigma Aldrich (USA), Xylitab 200 was donated by Danisco. 
$\mathrm{NaCl}$ was purchased from Merck (Germany). Acetate cellulose membrane with pore size $0.45 \mu \mathrm{m}$ was purchased from Sartorius Stedim Biotech (France).

\section{Preparation of the buccal films}

The wet film solution contained $3 \mathrm{~g}$ sodium alginate, $2 \mathrm{~g}$ Xilitab 200, $0.2 \mathrm{~g}$ tartrazine and $90 \mathrm{~g}$ purified water. The Xylitab and tartrazine were initially mixed with the water to obtain a clear solution, and under rapid stirring (magnetic stirrer) the sodium alginate was added slowly. This was important to minimise aggregation of the sodium alginate particles and to accelerate its dissolution. Initially, an unclear suspension was formed, which cleared with constant mixing and heating to $60^{\circ} \mathrm{C}$. The solution was then sonicated for 10 min to eliminate air bubbles. Subseqently, the solution was spread on glass plates $(35 \mathrm{~cm} \times$ $35 \mathrm{~cm}$ ) with an applicator (ZUA 2000; Zehnter, Switzerland), at different film thicknesses. The applicator settings of $1400 \mu \mathrm{m}$ and $1800 \mu \mathrm{m}$ were used. The glass plates with the films were then dried (SP-45; Kambič, Slovenia) at $65^{\circ} \mathrm{C}$ for $90 \mathrm{~min}$. The films obtained were cut into $2 \mathrm{~cm} \times 3 \mathrm{~cm}$ pieces.

\section{Tartrazine assay}

The tartrazine released from the films was measured with an ultraviolet-visible (UVVIS) spectrometer (8453 UV-visible spectrometer, Agilent, USA) at $425 \mathrm{~nm}$. The medium used was $0.9 \% \mathrm{NaCl}$ solution, with the same technique used for evaluation of the release samples.

\section{Release testing system}

The release flow cell consisted of two chambers made of poly(methyl methacrylate) that were separated by an acetate cellulose membrane with $0.45 \mu \mathrm{m}$ pore size. The chambers were positioned one on top of the other. The chamber dimensions were $2 \mathrm{~mm}$ smaller than those of the film, for width and length. The film was placed in the upper chamber and held fixed by the edges of the chamber. The film was positioned in the donor chamber, on top of the membrane. The release flow cell is illustrated on Fig. 1. The donor chamber only had an input for the medium to enter the chamber. The acceptor chamber had an input and an output for the medium. The main goal was to keep constant higher pressure for the medium in the donor chamber so that the film was pressed onto the membrane. Constant pressure was maintained also in the acceptor chamber to ensure the flow of the medium through the acceptor cell. Constant liquid pressure was achieved by applying constant hydrostatic pressure to the chamber.

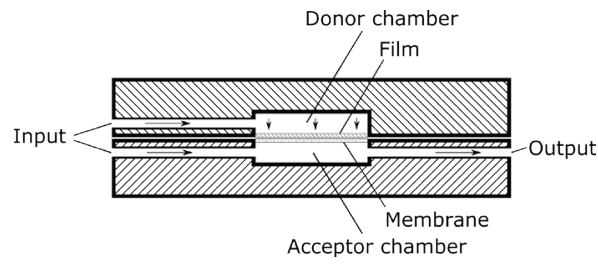

Fig. 1. The release flow cell design. 
The possibility of mass transfer resistance through the membrane and its effect on the drug release rate was taken into consideration. Therefore, a test film was dissolved in a 250 $\mathrm{mL}$ of dissolution media and the solution was poured at the donor side. At the acceptor side samples were taken and concentration of model drug was measured at different time points.

The dissolution medium in a beaker was positioned at a higher level than the flow cell. This beaker and the flow cell were connected by a tube and the medium flowed through the tube and the cell due to the height difference. During the emptying of the beaker, the liquid level decreased, and thus the pressure in the cell also decreased. To ensure constant hydrostatic pressure, a levelling device had been developed. This device determined the liquid level in the beaker, with the position of the beaker adjusted as the medium level decreased. Thus, as the liquid level in the beaker decreased, it had moved upwards by the same height difference. The precision of the levelling device was $0.5 \mathrm{~mm}$ in both directions.

The release rate system consisted of two separate levelling devices, one for each chamber (Fig. 2). The flow from the flow cell was collected in a reservoir that was constantly stirred by a magnetic stirrer. The system had a closed loop for the dissolution medium that flowed from the acceptor levelling beaker through the acceptor chamber into the reservoir. From the reservoir, the medium was pumped back to the acceptor levelling beaker using a peristaltic pump (503 S; Watson Marlow, England). The set-up of the release rate system

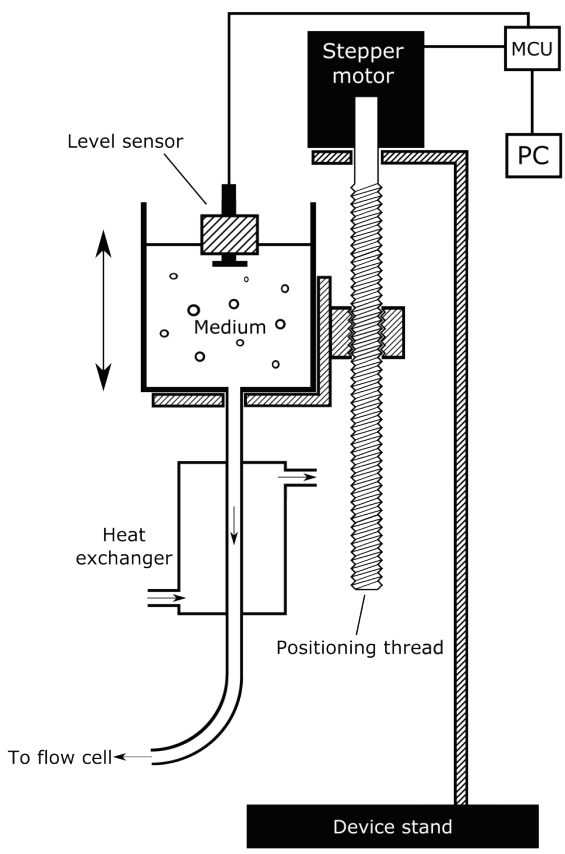

Fig. 2. The constant levelling device for the control of the hydrostatic pressure delivered to the donor chamber. MCU - microcontroller unit, PC - personal computer. 
is shown in Fig. 3. The pump for the reservoir was started when the medium level in the levelling beaker reached its lowest, and it was stopped when the levelling beaker was full again. The total volume of medium at the start was $250 \mathrm{~mL}$.

The medium that flowed through the donor chamber was always fresh and was not in the closed-loop system. The pressure applied to the donor chamber was higher than that for the acceptor chamber. However, the medium flow through the acceptor chamber was greater than of the donor chamber, due to the flow resistance of the membrane filter. The flow through the acceptor chamber was pre-set and kept constant, whereas the flow from the donor chamber changed with time, due to the dissolving of the film matrix. Thus, the dissolving of the film resulted in decreased flow resistance, although only for the medium in the donor chamber. Pressure in the donor chamber was about 1000 Pa greater than pressure in the acceptor chamber. This pressure difference was important for the achievement of tight contact between the film and the supporting membrane. Because the acetate cellulose membrane does not exhibit the same properties as human mucosa, the alginate films do not adhere to it. Therefore, the pressure difference maintained the film's position and pushed the film against the membrane.

To maintain the desired temperature of the dissolution medium $\left(37 \pm 0.5^{\circ} \mathrm{C}\right)$, a heat exchanger was used for the tubes through which the dissolution medium entered the flow cell. The temperature was thus controlled via a temperature sensor inside the acceptor chamber. The medium that carried the dissolved film components was collected in the reservoir, from which samples were taken. These sampling points were not time-based, but were event-based. Each time the acceptor chamber beaker became empty, a $10 \mathrm{~mL}$ sample was taken from the reservoir, with the sample volume replaced by fresh dissolution medium (de-aerated prior to use). The presence of air bubbles could cause fluctuation in the flow and pressure inside the flow cell. All of the temperature data and movements

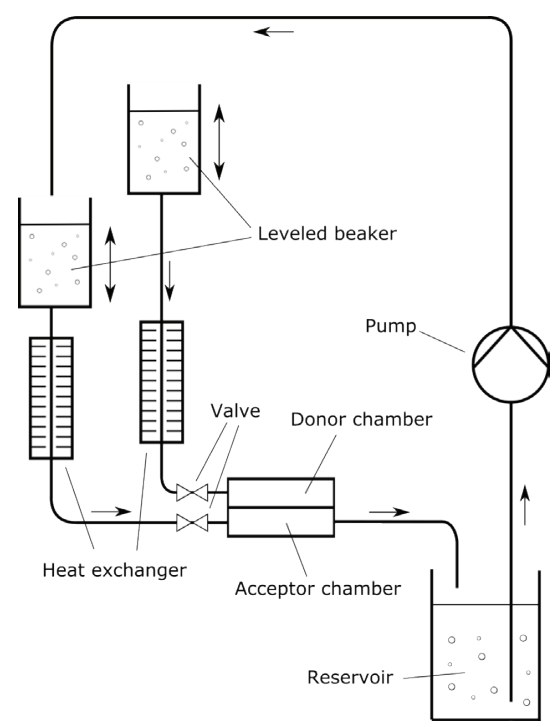

Fig. 3. Schematic depiction of the release system used. 
of the levelling device were collected for further evaluation. The whole process, including the sampling, was controlled through a computer, to minimise human error.

Each film used in the release process was precisely weighed before being placed carefully in the centre of the release cell. The film was covered by a membrane filter and the second cell block of the set-up, with the blocks tightened together firmly by four bolts. The flow cell containing the film was then fitted with the tubes and positioned in the stand (Fig. 2), which held the cell and the outlet tube at the same height at all times. The height difference between the outlet tube, the medium level, and the flow cell defined the hydrostatic pressure in the cell.

In setting up, $250 \mathrm{~mL}$ dissolution medium was poured into the reservoir, and before the start of the release process, all of the tubes and both levelling beakers were filled with this dissolution medium. The medium flow was blocked using two valves, with the release process started by opening both of the valves, which also triggered the computer software for the data capture. The samples were taken from the reservoir automatically using an autosampler, and further analysed using a UV-VIS spectrometer. All parts of the release testing system were designed and built by our research team.

\section{Initial experiments}

The first experiments were carried out to determine the importance of the wetting of the film from both sides during its release. In this situation, the film placed in the flow cell was isolated from the donor chamber by being covered with aluminium foil. This way it was no longer in contact with the medium on the donor (upper) side. Thus, the film swelled and dissolved only from the medium that was flowing through the acceptor chamber. Here, the donor chamber was filled with the medium as for the other release tests, whereby the hydrostatic pressure of the medium pressed the aluminium foil and the film onto the membrane. This maintained all of the parameters the same as in the other release tests, to only define the effects of wetting of the film on one side only.

\section{Variables in the release tests}

The second series of release tests for these films included the combinations of changes in the measurement conditions, for the medium flow through the acceptor chamber $(19,25$ $\left.\mathrm{g} \mathrm{min}^{-1}\right)$, the difference in height between the donor chamber medium level and the flow cell $(333,443 \mathrm{~mm})$, and the mass of the films $(40,60 \mathrm{mg})$. The experimental designs for these conditions are given in Table I for the definition of the two factorial levels $(-1,1)$ and the three factors $(A, B, C)$. Each of the eight conditions was repeated three times, to assess the

Table I. Definition of the experimental design for the two factorial levels $(-1,1)$ and the three factors $(A, B, C)$

\begin{tabular}{cccc}
\hline Condition & Medium flow $\left(\mathrm{mL} \mathrm{min}^{-1}\right)$ & Position of donor beaker $(\mathrm{mm})$ & Film mass $(\mathrm{mg})$ \\
\hline & A & B & C \\
-1 & 19 & 333 & 40 \\
1 & 25 & 443 & 60 \\
\hline
\end{tabular}


reproducibility. The mean release profiles and the coefficient of variation $(\mathrm{CV})$ were calculated. This experimental design was used to study which factor had greatest effects on the release process, and to determine whether the release process was driven by the film properties and was not a result of the variability of the device settings. The liquid flow rate in the acceptor chamber was selected by two factors. The first reason was to mimic the blood flow rate in the buccal veins. Squier et. al. measured buccal flow rate $20.3 \mathrm{~mL} \mathrm{~min}{ }^{-1}$ per $100 \mathrm{~g}$ of tissue (18). A second reason was to achieve sink condition on the acceptor side and prevent false time-delayed release detection.

\section{Discrimination ability of the method}

To assess the discrimination ability of the method, the dissolution profiles of the experiments varying in one parameter were compared. The comparison was done according to the international guidelines with the model-independent approach. The release profiles included percent of released tartrazine at times 5, 10, 15, 20, 25, 30, 35, 45, 60 and 80 minutes. The similarity factor $\mathrm{f} 2$ was calculated. Only one measurement was considered after $85 \%$ release of both instances. The $90 \%$ confidence interval (CI) for $\mathrm{f} 2$ was calculated by bootstrapping $(n=5000)$. Release profiles were accepted as similar if the lower CI of $\mathrm{f} 2$ was higher than 50. All calculations were performed using Excel add-in DDSolver (19).

\section{RESULTS AND DISCUSSION}

Testing of the mass transfer resistance showed that the membrane does not affect the model drug mass transfer to the acceptor chamber. Concentration increase did linearly correlate with the flow rate of the transferred media trough the membrane. Therefore, it seens reasonable to assume that the membrane does not affect the release profiles in a nonconstant manner and is appropriate for the release testing. Linear increase of the tartrazine amount in the reservoir is depicted in Fig. 4.

The release tests, where only the acceptor side of the film was wetted, provided some interesting data. With the inclusion of the aluminium foil on the donor side, the film

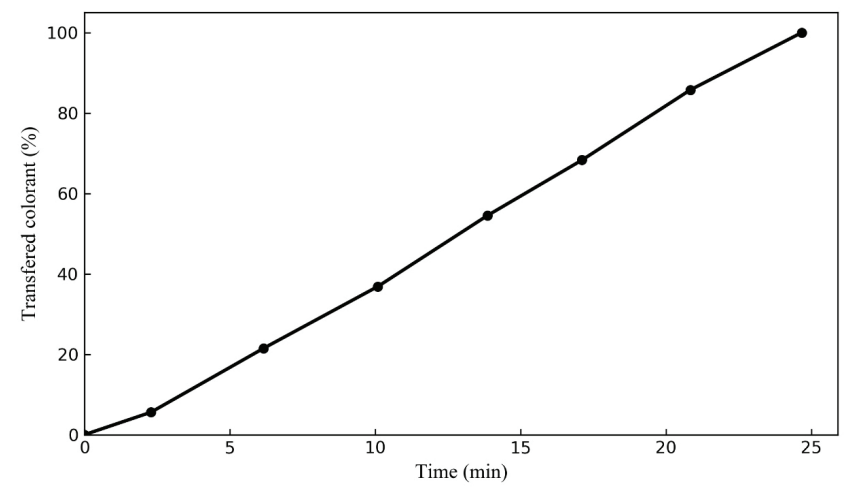

Fig. 4. Permeation of the film solution through the membrane into the acceptor compartment. 


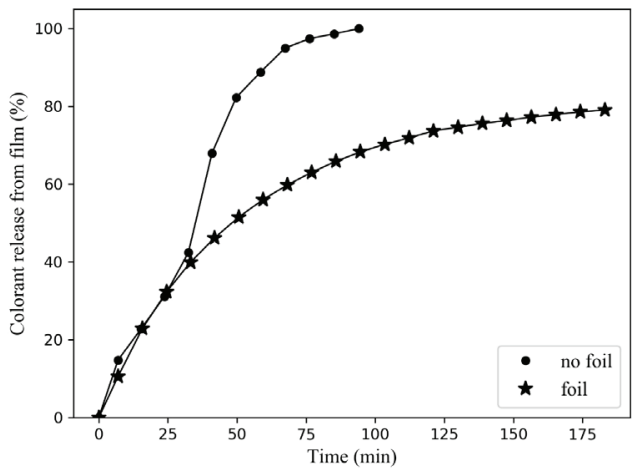

Fig. 5. Representative data for the release profiles of two films, with (foil) and without aluminium foil (no foil).

swelled only from the acceptor side which prolonged the release time of the incorporated colorant. Due to the fixed cell geometry, the colorant was released from a constant surface area. In the release profiles shown in Fig. 5, it can be seen that these two release profiles (i.e., \pm aluminium foil) started similarly, and remained similar up to $33 \mathrm{~min}$. At that time, the film colorant release rate without the aluminium foil increased more rapidly to reach $100 \%$ release at $94 \mathrm{~min}$. The film isolated with the aluminium foil continued to dissolve with a slower colorant release rate. There are no inflection points on this colorant release curve with the aluminium foil, and it took $183 \mathrm{~min}$ to release $80 \%$ of the colorant. After that time, the process was stopped. With the film wetted only from one side, it was not completely dissolved even after twice the release time compared to the film without aluminium foil, and wetted on both sides. Release profile comparison analysis showed significant differences between profiles depicted in Fig. 5. A calculated model-independent similarity factor value is 29.9. Therefore, the wetting of the film on the donor side had a significant impact on the release rate.

Fig. 6 illustrates the data from all of the release experiments across eight different designs. All of these profiles followed specific sigmoid curves. Here, each profile started with almost constant release of the colorant, which then increased at some point during its release. This is visible as inflections in these curves.

During this monitoring of the colorant release, the flow of the medium from the donor chamber to the acceptor chamber was tracked according to the movement of the levelling beaker. At the beginning of these release processes, none of the medium was lost from the beaker, which meant that no medium flowed from the donor to the acceptor chamber. Thus, in the beginning, the film represents a barrier for the medium to flow from the donor chamber to the acceptor chamber. Then the donor beaker flow rate started to increase with time in a non-linear manner. Here, it was expected that the increase in the donor chamber flow rate would correlate with the increase in the colorant release rate. However, the flow rate from the donor to the acceptor chamber did not start to increase until $100 \%$ of the colorant had been released, as illustrated in Fig. 7.

These release profiles can be divided into three parts. In the first part, there was a semi-constant release rate for the colorant, due to the medium that flowed through the 


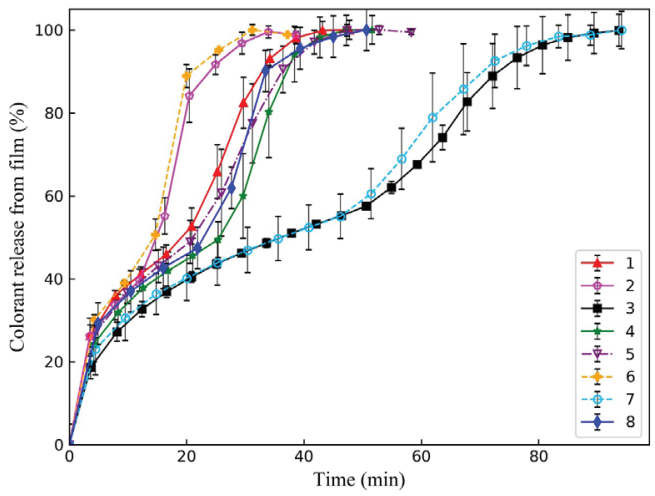

Fig. 6. Release profiles for all of the eight conditions (Table II) examined here.

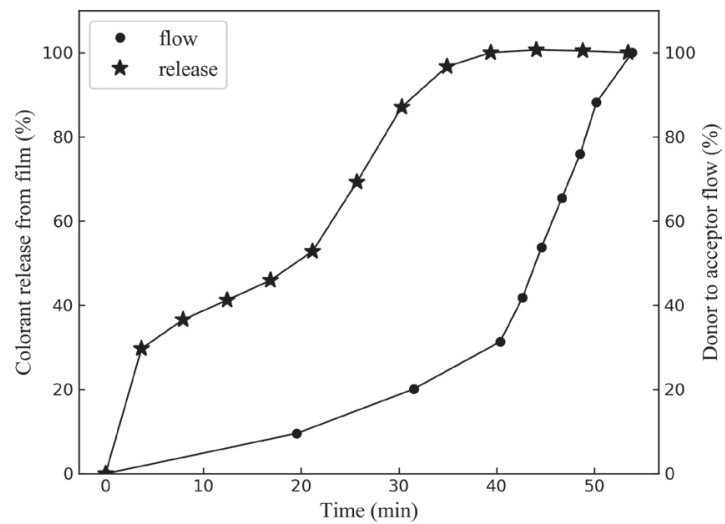

Fig. 7. Representative data for the tartrazine release rates compared to the flow of the medium from the donor chamber to the acceptor chamber.

acceptor chamber to wet the film on one side, through the membrane. The film then swelled and the colorant was constantly released and flushed into the acceptor reservoir. During this time, the film has been also swelling on the top, in the donor chamber, but because of the barrier that was formed, only the colorant from the bottom of the film was released into the acceptor chamber. In the second part, the medium penetrated more into the film and softened this barrier. The film then swelled to the point that it was pushed through the membrane by the medium pressure in the donor chamber. The dissolved film content was thus transferred to the acceptor chamber, where the medium flow flushed it into the reservoir. At this point, the release rate of the colorant was increased, which is visible as an inflection in the profile of the colorant release curve. During this release period the film was penetrated and its resistance as a barrier to the flow from donor to acceptor chambers decreased. This resulted in increased flow rate from donor to acceptor, and con- 
sequently, the film moved through the membrane and there was full release of the colorant from the film. The speed of this process was a reflection of the film's properties. Using this type of release design allows observation of the swelling properties of the polymer. The inflection point on the release profile may represent the time at which the film is completely swollen. This is advantageous over the other release methods. Also, the acetate cellulose membrane could be easily changed by the etched mucosa for ex vivo studies and providing better insight into in vivo conditions.

The reproducibility of the experiments was satisfactory. The CV was less than $20 \%$ for time points $15 \mathrm{~min}$ or less in all cases, except for the experiment 7 at $5 \mathrm{~min}(\mathrm{CV}=26 \%)$. The CV was $10 \%$ or less for time points higher than $15 \mathrm{~min}$, with slightly higher variability observed only in experiment 7. Nevertheless, the CV was never higher than $15 \%$.

The results from the experimental design are given in Table II, where the time needed for $80 \%$ of colorant release was used as the reference point for comparisons across the eight conditions. The factor effects were calculated using a factor analysis approach, whereby the biggest effect on film release time was the mass of the film. In the present case, the mass was directly proportional to the film thickness, because the films were cut to the same size of $2.0 \mathrm{~cm} \times 3.0 \mathrm{~cm}$, and thus we can interpret this mass factor as a film thickness factor. There were also large effects on the release time according to the position of the donor beaker. This was reduced in this design by $25 \%$ from the original setting (i.e., from $443 \mathrm{~mm}$ to $333 \mathrm{~mm}$ ), which was defined as a significantly important factor. Hydrostatic pressure in a donor chamber is normally not changed between release tests. Here, it is shown that it is important to keep the height ratio constant for comparisons between formulations. Surprisingly, the factor of the flow rate through the acceptor chamber was particularly low. This is a good result because variations in the flow will not influence the discriminatory properties of this method.

Table II. Design of the experiments carried out, and their factor effects on the film release rates

\begin{tabular}{ccccccccc}
\hline $\mathrm{n}$ & $\mathrm{A}$ & $\mathrm{B}$ & $\mathrm{C}$ & & & & & \\
\cline { 2 - 5 } & $\begin{array}{c}\text { Medium } \\
\text { flow } \\
\left(\mathrm{g} \mathrm{min}^{-1}\right)\end{array}$ & $\begin{array}{c}\text { Beaker } \\
\text { position } \\
(\mathrm{mm})\end{array}$ & $\begin{array}{c}\text { Film } \\
\text { mass } \\
(\mathrm{mg})\end{array}$ & $\mathrm{A} \times \mathrm{B}$ & $\mathrm{A} \times \mathrm{C}$ & $\mathrm{B} \times \mathrm{C}$ & $\mathrm{A} \times \mathrm{B} \times \mathrm{C}$ & $\begin{array}{c}t(80 \%) \\
(\mathrm{min})\end{array}$ \\
\hline 1 & 19 & 333 & 40 & -1 & -1 & 1 & 1 & 29 \\
2 & 19 & 433 & 40 & 1 & -1 & -1 & -1 & 20 \\
3 & 19 & 333 & 60 & -1 & 1 & -1 & -1 & 64 \\
4 & 19 & 433 & 60 & 1 & 1 & 1 & 1 & 34 \\
5 & 25 & 333 & 40 & 1 & 1 & 1 & -1 & 31 \\
6 & 25 & 433 & 40 & -1 & 1 & -1 & 1 & 19 \\
7 & 25 & 333 & 60 & 1 & -1 & -1 & 1 & 63 \\
8 & 25 & 433 & 60 & -1 & -1 & 1 & -1 & 32 \\
Effect & 0.5 & -20.5 & 23.5 & 1.0 & 1.0 & -10.0 & -0.5 & \\
\hline
\end{tabular}

$t(80 \%)$ - time needed for $80 \%$ tartrazine release from the film. 
Table III. Comparison of the release profiles

\begin{tabular}{ccccccc}
\hline Pair & A & B & C & & f2 & $\begin{array}{c}\text { Bootstrap median } \\
\text { f2 (90 \% CI })\end{array}$ \\
\cline { 2 - 4 } & $\begin{array}{c}\text { Medium flow } \\
\left(\mathrm{g} \text { min }^{-1}\right)\end{array}$ & $\begin{array}{c}\text { Beaker position } \\
(\mathrm{mm})\end{array}$ & $\begin{array}{c}\text { Film mass } \\
(\mathrm{mg})\end{array}$ & & & \\
\hline $1-1$ & 19 vs. 25 & 333 & 40 & 74.7 & $71.9(56.7-89.8)$ \\
$1-2$ & 19 vs. 25 & 433 & 40 & 63.5 & $63.5(51.4-73.3)$ \\
$1-3$ & 19 vs. 25 & 333 & 60 & 68.5 & $67.1(53.0-85.9)$ \\
$1-4$ & 19 vs. 25 & 433 & 60 & 64.0 & $63.1(50.2-74.8)$ \\
$2-1$ & 19 & 333 vs. 433 & 40 & & 29.6 & $29.4(26.9-32.6)$ \\
$2-2$ & 25 & 333 vs. 433 & 40 & & 33.6 & $33.6(31.0-36.8)$ \\
$2-3$ & 19 & 333 vs. 433 & 60 & & 31.8 & $31.7(28.5-35.1)$ \\
$2-4$ & 25 & 333 vs. 433 & 60 & & 30.6 & $30.6(27.7-33.3)$ \\
$3-1$ & 19 & 333 & 40 vs 60 & & 25.7 & $25.7(22.8-29.0)$ \\
$3-2$ & 19 & 433 & 40 vs 60 & & 24.5 & $24.5(22.4-26.9)$ \\
$3-3$ & 25 & 333 & 40 vs 60 & & 23.5 & $23.5(22.5-24.6)$ \\
$3-4$ & 25 & 433 & 40 vs 60 & 25.4 & $25.4(22.8-28.2)$ \\
\hline
\end{tabular}

There were, however, minor differences between the release profiles. When there was a higher flow rate, the release time was a little shorter. Comparing profiles 2 and 6 or 3 and 7 in Fig. 6, the effect of flow rate was smaller compared to the beaker position and film thickness. There was also a significant effect of the combined $\mathrm{B} \times \mathrm{C}$ factors. This indicated that it was potentially important to set the donor beaker position according to the film thickness to obtain the most discriminatory data. However, the major effects are related to the film properties, which are the main interest of this release method.

The calculated f2 similarity factors additionally confirm the above (Table III). The mass factor and the position of the donor beaker were significant parameters, considering the release profile comparison in these cases demonstrated significant differences. The 90 $\%$ CI of $\mathrm{f} 2$ factors was below 50 . Specifically, in the case of varying beaker position, the 90 $\%$ CIs of $\mathrm{f} 2$ across all comparisons were between 26.9 and 36.8, which corresponds to the average difference between 18 and $30 \%$. When film mass was varied, the $90 \%$ CI of $\mathrm{f} 2$ was between 22.4 and 29.0, which corresponds to the average difference between 25 and $35 \%$. In contrast, when the flow rate was varied, the lower CI of $\mathrm{f} 2$ was higher than 50 in all cases, thus accepting profiles as similar and the parameter as nonsignificant. The average difference of the release profiles obtained by varying the flow rate was within $10 \%$.

\section{CONCLUSIONS}

The drug release testing from a dosage form provides an insight into product performance and obtained data could further guide the formulation development process. A discriminatory release method to evaluate buccal films was thus investigated. The pre- 
sented method based on a flow cell is shown to be repeatable and suitable for release rate evaluation for this type of formulation. The factors with the greatest effects on the release method were followed here.

The factor with the greatest influence on the release rate shown was the film thickness, as a direct film property; this is good because it shows that the method should express differences between formulations. The second-largest effect is related to the donor chamber pressure, which can be easily controlled through the height of the levelling beaker. The medium flow rate also had a relatively minor effect on the release rate. This is beneficial for the reproducibility of the measurements. The importance of the wetting of the films from both sides was also discussed to be essential for the discriminatory release testing. This method thus provides further advantages over others. It could also be used for film formulations that are releasing the active ingredients from only one side. The drug release conditions of proposed release method do not reflect the in vivo conditions in detail, but the method was shown to be repeatable and discriminatory for evaluation of film formulations.

Acknowledgements. - We thank the Klaria Pharma Holding AB and Karessa Pharma AB for the founding of this study.

\section{REFERENCES}

1. A. Semalty, M. Semalty and U. Nautiyal, Formulation and evaluation of mucoadhesive buccal films of enalapril maleate, Indian J. Pharm. Sci. 72 (2010) 571-575; https://doi.org/0.4103/0250-474X.78522

2. Y. Sudhakar, K. Kuotsu and A. K. Bandyopadhyay, Buccal bioadhesive drug delivery - a promising option for orally less efficient drugs, J. Control. Release 114 (2006) 15-40; https://doi.org/10.1016/j. jconrel.2006.04.012

3. V. F. Patel, F. Liu and M. B. Brown, Advances in oral transmucosal drug delivery, J. Control. Release 153 (2011) 106-116; https://doi.org/10.1016/j.jconrel.2011.01.027

4. J. A. Nicolazzo, B. L. Reed and B. C. Finnin, Buccal penetration enhancers--how do they really work?, J. Control. Release 105 (2005) 1-15; https://doi.org/10.1016/j.jconrel.2005.01.024

5. S. Lindgren and L. Janzon, Prevalence of swallowing complaints and clinical findings among 50-79-year-old men and women in an urban population, Dysphagia 6 (1991) 187-192.

6. V. Prajapati, A. Chaudhari, A. Gandhi and P. Maheriya, Pullulan based oral thin film formulation of zolmitriptan: Development and optimization using factorial design, Int. J. Biol. Macromol. 107 (2017) 2075-2085; https://doi.org/10.1016/j.ijbiomac.2017.10.082

7. Z. Cui and R. J. Mumper, Bilayer films for mucosal (genetic) immunization via the buccal route in rabbits, Pharm. Res. 19 (2002) 947-953.

8. N. M. G. Almeida, R. Lima, T. F. R. Alves, M. de A. Rebelo, P. Severino, M. V. Chaud, N. M. G. Almeida, R. Lima, T. F. R. Alves, M. de A. Rebelo, P. Severino and M. V. Chaud, A novel dosage form for buccal administration of bupropion, Braz. J. Pharm. Sci. 51 (2015) 91-100; https://doi. org/10.1590/S1984-82502015000100010

9. V. Garsuch and J. Breitkreutz, Novel analytical methods for the characterization of oral wafers, Eur. J. Pharm. Biopharm. 73 (2009) 195-201; https://doi.org/10.1016/j.ejpb.2009.05.010

10. S. Karki, H. Kim, S.-J. Na, D. Shin, K. Jo and J. Lee, Thin films as an emerging platform for drug delivery, Asian J. Pharm. Sci. 11 (2016) 559-574; https://doi.org/10.1016/j.ajps.2016.05.004

11. J.-M. Cardot, B. Roudier and H. Schütz, Dissolution comparisons using a Multivariate Statistical Distance (MSD) test and a comparison of various approaches for calculating the measurements 
of dissolution profile comparison, AAPS J. 19 (2017) 1091-1101; https://doi.org/10.1208/s12248-0170063-y

12. Y. Xia, F. Chen, H. Zhang and C. Luo, A new method for evaluating the dissolution of orodispersible films, Pharm. Dev. Technol. 20 (2015) 375-379; https://doi.org/10.3109/10837450.2014.882936

13. A. N. ElMeshad and A. S. El Hagrasy, Characterization and optimization of orodispersible mosapride film formulations, AAPS PharmSciTech 12 (2011) 1384-1392; https://doi.org/10.1208/s12249011-9713-z

14. R. Krampe, D. Sieber, M. Pein-Hackelbusch and J. Breitkreutz, A new biorelevant dissolution method for orodispersible films, Eur. J. Pharm. Biopharm. 98 (2016) 20-25; https://doi.org/10.1016/j. ejpb.2015.10.012

15. R. Laitinen, J. Räty, K. Korhonen, J. Ketolainen and K.-E. Peiponen, Reflectometric monitoring of the dissolution process of thin polymeric films, Int. J. Pharm. 523 (2017) 127-132; https://doi. org/10.1016/j.ijpharm.2017.03.028

16. A. Adrover and M. Nobili, Release kinetics from oral thin films: Theory and experiments, Chem. Eng. Res. Des. 98 (2015) 188-201; https://doi.org/10.1016/j.cherd.2015.04.016

17. A. Adrover, A. Pedacchia, S. Petralito and R. Spera, In vitro dissolution testing of oral thin films: A comparison between USP 1, USP 2 apparatuses and a new millifluidic flow-through device, Chem. Eng. Res. Des. 95 (2015) 173-178; https://doi.org/10.1016/j.cherd.2014.10.020

18. C. A. Squier and D. Nanny, Measurement of blood flow in the oral mucosa and skin of the rhesus monkey using radiolabelled microspheres, Arch. Oral Biol. 30 (1985) 313-318.

19. Y. Zhang, M. Huo, J. Zhou, A. Zou, W. Li, C. Yao and S. Xie, DDSolver: An add-in program for modeling and comparison of drug dissolution profiles, AAPS J. 12 (2010) 263-271; https://doi. org/10.1208/s12248-010-9185-1 